\title{
Electronic portal imaging registration in breast cancer radiotherapy verification: Analysis of inter-observer agreement among different categories of health practitioners
}

\author{
B. A. JERECZEK-FOSSA ${ }^{1,6}$, L. SANTORO ${ }^{4}$, S. P. COLANGIONE ${ }^{1,6}$, L. MORSELLI ${ }^{6}$, C. FODOR ${ }^{1}$, B. VISCHIONI ${ }^{5}$, D. ROZZA ${ }^{1}$, A. LEPPA ${ }^{1}$, R. CAMBRIA ${ }^{2}$, \\ M. C. LEONARDI ${ }^{1}$, A. MORRA ${ }^{1}$, G. BARONI ${ }^{5,7}$, S. ZURRIDA ${ }^{3,6}$, R. ORECCHIA ${ }^{1,5,6}$
}

${ }^{1}$ Dept. of Radiation Oncology, European Institute of Oncology, Milan, Italy; ${ }^{2}$ Dept. of Medical Physics, European Institute of Oncology, Milan, Italy; ${ }^{3}$ Breast Diagnosis and Surgery Unit, Senology Division; European Institute of Oncology, Milan, Italy; ${ }^{4}$ Dept. of Epidemiology and Biostatistic, European Institute of Oncology, Milan, Italy; ${ }^{5}$ Centro Nazionale di Adroterapia Oncologica, Pavia, Italy; ${ }^{6}$ University of Milan, School of Medicine, Milan, Italy; ${ }^{7}$ Department of ICT and Bioengineering, Politecnico di Milano, Milan, Italy

${ }^{\star}$ Correspondence: barbara.jereczek@ieo.it

Received August 22, 2012 / Accepted October 24, 2012

\begin{abstract}
Electronic portal imaging (EPI) is commonly used to identify and correct for inter-fraction variability in tangential breast irradiation. Based on the institutional policy, EPI registration is performed by either radiation oncologist or therapist. Little data is available on the inter-observer agreement in EPI registration among different health practitioners. The aim of our study was to analyze inter-observer agreement among radiation oncologists and therapists in the evaluation of EPI for breast cancer radiotherapy verification. EPI data of 40 patients treated with tangential fields were independently reviewed by a radiation oncologist (on-line, just before treatment) and off-line by junior and senior therapists. Displacement of each EPI image with respect to the digital reconstructed radiographs (DRRs) was quantified using manual EPI registration based on bony marks with the corresponding DRRs. Agreement between observers was evaluated using weighted Cohen's Kappa statistics. In 95\% out of 720 EPI-DRR comparisons, the EPI-DRR misalignment was $<5 \mathrm{~mm}$. The difference between observers was $<2 \mathrm{~mm}$ in 666 (92.5\%) out of all 720 delta values. High inter-observer agreement was found, with weighted Cohen's Kappa values attesting evaluation overlaps ranging from moderate (among therapists) to almost perfect (among radiation oncologist and therapists). The high agreement among the observers demonstrated the precision of breast localization using EPI. These findings suggest that routine EPI-based patient set-up verification in breast cancer radiotherapy can be safely entrusted to trained therapists (supervision should be assured based on the local tasks definition). Our study might be useful in quality assurance and in the optimization of workload in the radiotherapy departments. They might allow for wider implementation of complex and evolving radiotherapy technologies.
\end{abstract}

Key words: EPI, breast cancer radiotherapy, radiation oncologist, radiation therapist, inter-observer agreement, Kappa statistics

Electronic portal imaging (EPI) represents a gold standard for patient set-up verification in breast cancer radiotherapy $[1,2]$. It has also gained increasingly importance for intensity modulated radiotherapy (IMRT) dose verification, both pretreatment and in vivo [3], as well as for motion tracking (EPI acquired in cine mode) [4]. In many departments, EPI derived images evaluation is still performed by radiation oncologists [5]. However, in daily clinical practice, the majority of treatment geometry verification procedures images are entrusted to therapists. This turns out to be particularly important when many image-guided therapy sessions are performed daily, requiring the use of complex set-up verification techniques (cone beam computer tomography, ultrasound imaging, etc.) and mandatory radiation oncologist supervision [6-8]. Careful verification of the EPI-based set-up evaluations performed by therapists against the one featured by radiation oncologists is crucial, as a way to ensure an equivalent quality level in treatment geometry verification. The aim of this study was to verify the inter-observer evaluation agreement among radiation oncologists and therapists, when reviewing EPI images acquired for patient set-up verification in breast cancer external beam radiotherapy. Based on this study results the decision 


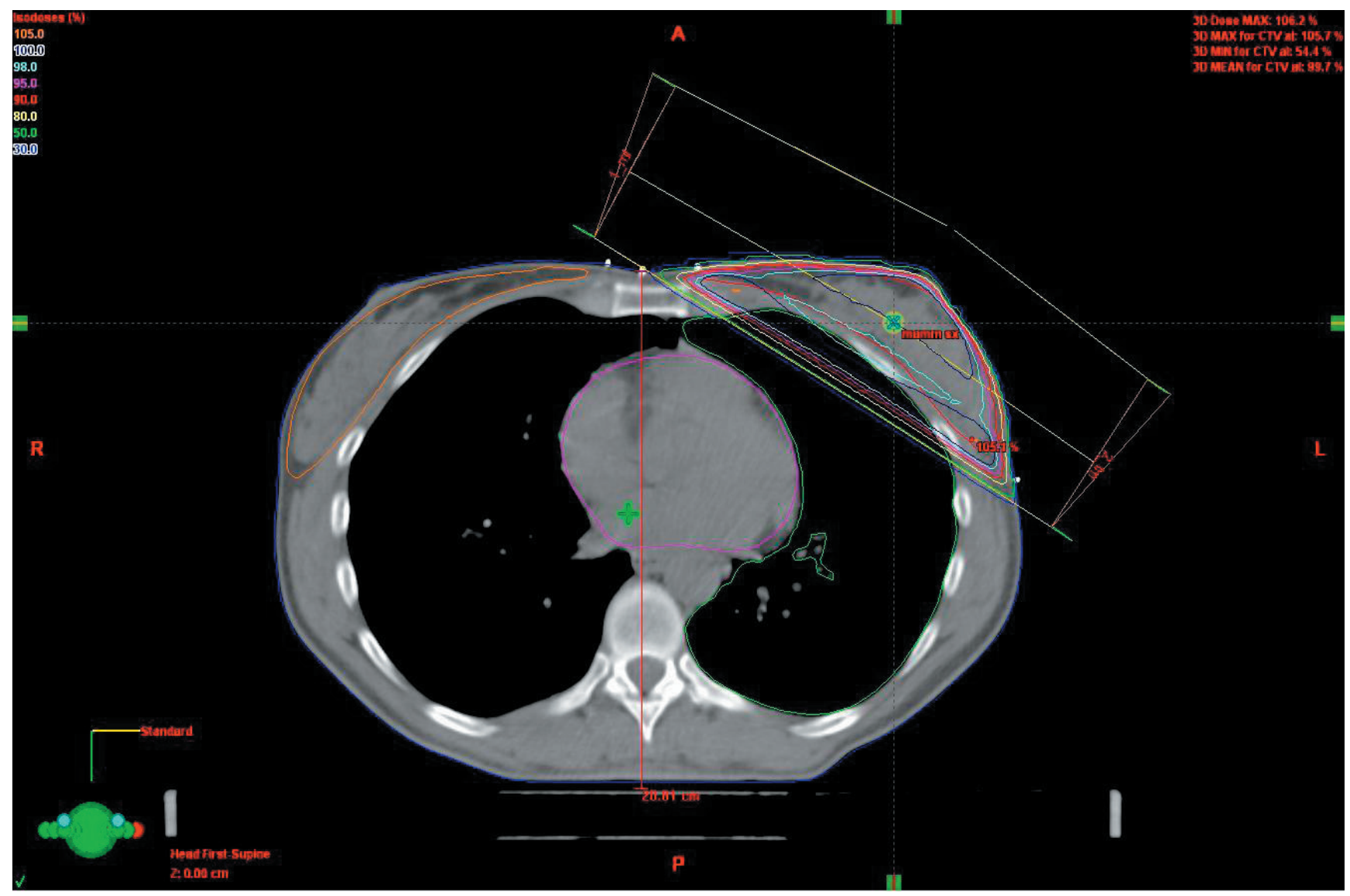

Figure 1. Isodose distribution in the three-dimensional conformal radiotherapy for breast cancer.

on the task division in the set-up verification institutional protocols were planned.

\section{Materials and methods}

Inclusion criteria. The patient inclusion criteria for this study were as follows: non-metastatic breast cancer (both invasive and in situ tumors were eligible), breast surgery (including breast conserving surgery and mastectomy), indication for postoperative irradiation with tangential fields only, availability of routine set-up verification data based on the institutional EPI protocol. The patients with supraclavicular or internal mammary chain irradiation were not eligible for this study.

Radiotherapy protocol. Postoperative breast radiotherapy is performed in our Department using 3-dimensional conformal radiotherapy (3DCRT) (figure 1, online only).

The conventional regimen after breast conserving surgery includes whole breast radiotherapy to the dose of $50 \mathrm{~Gy}$ in 25 fractions prescribed at the International Commission of Radiation Units (ICRU) point [9] and a boost to the tumor bed administrated by direct electron field to $10 \mathrm{~Gy}$ in 5 fractions. More recently, hypofractionated schedules have been introduced $[10,11]$.
All patients undergo computer tomography (CT) based treatment planning with $3 \mathrm{~mm}$ slicing in supine position, with both arm abducted. Immobilization is achieved by means of customized devices that provide support to head, arms and thorax. The Eclipse 8.6 treatment planning system (Varian, Palo Alto, USA) is employed. Clinical target volume (CTV) is contoured by a radiation oncologist on each CT slice. A $5 \mathrm{~mm}$, latero-lateral and $15 \mathrm{~mm}$ cranio-caudal margins are added to the CTV, for planning target volume (PTV) generation, accounting for set-up inaccuracies and respiratory motion. This latter has been quantified by optical tracking systems to introduce slight geometric inaccuracies (along the main anatomical axes) [12], thus supporting the adequacy of a free-breathing approach and the applied safety margin accounting for breathing induced uncertainties. Organs at risk (heart, lung, contra-lateral breast) are identified and dose volume histograms (DVHs) are elaborated for each patient.

The treatment consists in two photon tangential fields of $6 \mathrm{MV}$ energy. Beams are produced by our CLINAC $600 \mathrm{C} / \mathrm{D}$ (Varian, Palo Alto, USA) equipped with the Millennium ${ }^{\circledR}$ multileaf collimator (52 leaf; field size 26 x $40 \mathrm{~cm}$; leaf widths $10 \mathrm{~mm}$ ) and with the Electronic Portal Vision ${ }^{\circledR}$ v. 5.0. 

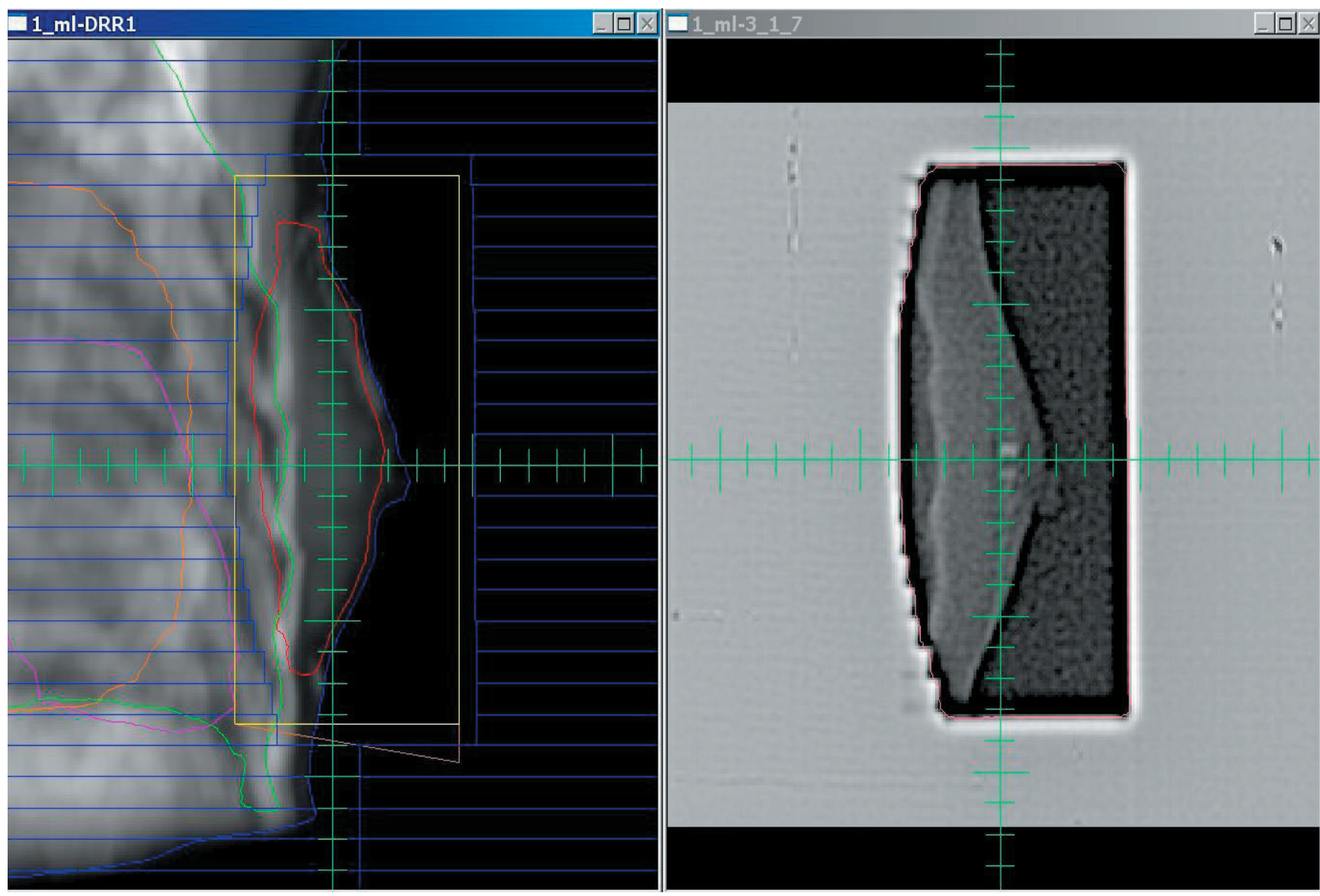

Figure 2. Digitally reconstructed radiograph (left) and corresponding electronic portal imaging of the medio-lateral tangential breast field.

Individual multi-leaf collimator shielding is used, in order to reduce the dose to the normal tissue. Collimator rotation is introduced, if needed, to reduce the amount of lung volume included in the irradiation field. Compensating filters are employed to avoid inhomogeneities in dose distribution caused by breast morphology. The isocenter is set in correspondence of the middle axis of the tangential field. For patient set-up, 4 reference skin marks are tattooed: 2 anterior midline landmarks at the level of isocenter and caudal border of the field and 2 lateral mid-axillary points. Patient positioning is obtained by aligning the sagittal and lateral laser beam references on the tattooed skin marks. All fields are administered once a day, 5 days a week. The radiation therapy is delivered on an outpatient basis. In vivo dosimetry and EPI set-up verification is performed on the first day of the treatment.

EPI protocol. The institutional EPI protocol in our Department envisages EPI-based verification on the first treatment day. After patient positioning based on manual laser alignment, EPI images are acquired and on-line verification is performed by a radiation oncologist. EPI images of medio-lateral (ML) and latero-medial (LM) tangential fields are compared and manually matched to their correspond- ing digitally reconstructed radiographs (DRRs) generated from the CT used for treatment planning (figure 2, online only). Matching is based on manual registration using bony anatomy; central lung distance is also measured and compared to the DRRs value. The resulting misalignments are automatically reported along antero-posterior (AP), latero-lateral (LL) and cranio-caudal (CC) directions. Mismatches are compensated by acting on the treatment couch by a radiation therapist.

The institutional action level has been established as follows: in case of AP or CC misalignment lower or equal than the safety margin between CTV and PTV, the first radiotherapy fraction is delivered to the patient and no further verification is required during the whole radiotherapy course. Conversely, if detected misalignments are between 6 and $10 \mathrm{~mm}$, couch based correction is performed and further EPI verification (and correction) on treatment days 2 and 3 is required. On the treatment day 4 , the mean values of the $3 \mathrm{EPI}$ measurements (performed on days 1,2 and 3) in each direction are calculated. In case the resulting mean misalignments are below $5 \mathrm{~mm}$ along any anatomical axis, the patient is treated without any further verification and correction. If mean misalignment (along any anatomical axis) is turn 
out to be between 6 and $10 \mathrm{~mm}$, the set-up is corrected by the mean value and verified by EPI on the day 4 and subsequently, the set-up is corrected at all following therapy fractions by the same mean value. For misalignment detected on day 1 higher than $10 \mathrm{~mm}$, the patient is re-positioned and new EPI verification is undertaken; in case of persistence of a misalignment above $10 \mathrm{~mm}$, new CT simulation and treatment planning are performed.

Study protocol. One junior therapist and one senior therapist with 11 year experience were involved in the study. Both of them received a brief but exhaustive training by radiation oncologists. For the purpose of this study, the set of the 40 first-day-EPI images were reviewed against the DRRs off-line independently by the 2 therapists. The verification EPI-images (see the above institutional protocol including day 2, 3 and 4 EPI acquisition in patients with the first-day misalignment between 6 and $10 \mathrm{~mm}$ ) were not used for the inter-observer agreement analysis. Time trends as well as random and systematic deviations were not subject of this study.

The reference for the assessment of therapists EPI-DRR interpretation was the EPI-DRR evaluation performed by a radiation oncologist at the first irradiation session.

Statistical analysis. Measured EPI-DRR misalignments were summarized by mean and standard deviation calculated grouping together the 3 observers as well as considering them individually. Agreement between observers (inter-rater agreement) was evaluated by means of the weighted Cohen's Kappa, with weights given by the magnitude of the difference between observers. The interpretation of the weighted Cohen's Kappa followed the Landis and Koch scoring system [13]. Weighted Cohen's Kappa coefficient $<0$ means poor agreement between observers, whereas coefficient ranges of $0.0-0.2,0.21-0.4,0.41$ $-0.6,0.61-0.8$ and $0.81-1.0$ represent light, discrete, moderate, substantial and almost perfect agreement, respectively.

\section{Results}

Study population. The EPI data of 40 consecutive early breast cancer patients satisfying the inclusion criteria and treated between the $1^{\text {st }}$ and $31^{\text {st }}$ July 2010 at the Division of Radiotherapy of the European Institute of Oncology, Milan, Italy were included in the study. The mean age of the patients was 55 years (range, $34-84$ years). All patients underwent postoperative radiotherapy after breast conserving surgery (35 patients) or mastectomy (5 patients). Right and left breast was treated in 22 and 18 cases, respectively. Thirty seven patients had invasive cancer and in 3 cases - in situ lesions were treated. All patients gave a written informed consent for the postoperative irradiation.

EPI data. A total of 80 EPI images were analyzed (ML and LM EPI images for 40 patients) giving rise to 240 EPI-DRR evaluations for the pool of 3 observers. Since each observer was asked to report misalignments along the 3 anatomical axes, a total of 720 EPI-DRR measurements were analyzed.

As far as ML projection is concerned, a mean AP EPI-DRR misalignment registered by a radiation oncologist, senior and junior technologist was $-1.87 \mathrm{~mm},-2.27 \mathrm{~mm}$ and $-2.0 \mathrm{~mm}$, respectively (tab. 1). Based on the misalignment reported by a radiation oncologist, senior and junior technologist 1 , 2 and 1 patients would need further EPI verification (due to a misalignment with DRR $>5 \mathrm{~mm}$, see above the action level institutional protocol), respectively. The difference in measurements between observers (120 delta values) was $3,4,5$, and $6 \mathrm{~mm}$ in 5, 1, 2 and 1 case, respectively, in all other 111 cases (92.5\%) the difference was $<2 \mathrm{~mm}$.

A mean CC EPI-DRR misalignment on the ML projection registered by a radiation oncologist, senior and junior technologist was $1.45 \mathrm{~mm}, 1.85 \mathrm{~mm}$ and $1.27 \mathrm{~mm}$, respectively. Based on the misalignment reported by a radiation oncologist, senior and junior technologist 3, 4 and 3 patients would need further EPI verification (due to a misalignment with DRR > $5 \mathrm{~mm}$ ), respectively. The difference in measurements between observers (120 delta values) was 3, 4 and $5 \mathrm{~mm}$ in 6, 2 and 4 cases, respectively. In all other 108 cases (90\%) the difference was $<2 \mathrm{~mm}$.

A mean LL EPI-DRR misalignment on the ML projection registered by a radiation oncologist, senior and junior technologist was $0.65 \mathrm{~mm}, 0.57 \mathrm{~mm}$ and $0.62 \mathrm{~mm}$, respectively.

Table 1. Mean misalignment values between EPI and reference DRR, by observer for all patients $(n=40)$

\begin{tabular}{|c|c|c|c|c|c|c|c|c|}
\hline & \multicolumn{2}{|c|}{$\begin{array}{l}\text { Radiation oncologist } \\
\qquad(\mathrm{n}=40)\end{array}$} & \multicolumn{2}{|c|}{$\begin{array}{l}\text { Senior radiation therapist } \\
\qquad(\mathrm{n}=40)\end{array}$} & \multicolumn{2}{|c|}{$\begin{array}{l}\text { Junior radiation therapist } \\
\qquad(\mathrm{n}=40)\end{array}$} & \multicolumn{2}{|c|}{$\begin{array}{l}\text { All observers } \\
(\mathrm{n}=120)\end{array}$} \\
\hline & $\begin{array}{l}\text { Mean, mm } \\
\quad(S D)\end{array}$ & $\begin{array}{l}\text { Range } \\
(\mathrm{mm})\end{array}$ & $\begin{array}{l}\text { Mean, mm } \\
\quad(\text { SD) }\end{array}$ & $\begin{array}{l}\text { Range } \\
(\mathrm{mm})\end{array}$ & $\begin{array}{l}\text { Mean, mm } \\
\quad(\mathrm{SD})\end{array}$ & $\begin{array}{l}\text { Range } \\
(\mathrm{mm})\end{array}$ & $\begin{array}{l}\text { Mean, mm } \\
\quad(\mathrm{SD})\end{array}$ & $\begin{array}{l}\text { Range } \\
(\mathrm{mm})\end{array}$ \\
\hline ML AP & $-1.87(2.45)$ & -7 to 3 & $-2.27(2.60)$ & -9 to 2 & $-2.0(2.50)$ & -8 to 4 & $-2.0(2.50)$ & -9 to 4 \\
\hline ML CC & $1.45(2.70)$ & -2 to 8 & $1.85(2.69)$ & -2 to 8 & $1.27(2.65)$ & -3 to 7 & $1.52(2.67)$ & -3 to 8 \\
\hline ML LL & $0.65(2.00)$ & -4 to 4 & $0.57(2.21)$ & -4 to 6 & $0.62(2.00)$ & -4 to 4 & $0.62(2.05)$ & -4 to 6 \\
\hline LM AP & $-2.15(2.47)$ & -7 to 3 & $-2.25(2.60)$ & -7 to 3 & $-2.17(2.75)$ & -8 to 4 & $-2.2(2.59)$ & -8 to 4 \\
\hline LM CC & $1.50(2.86)$ & -5 to 9 & $1.65(3.17)$ & -5 to 8 & $1.40(2.79)$ & -5 to 7 & $1.52(2.92)$ & -5 to 9 \\
\hline LM LL & $0.75(2.54)$ & -6 to 5 & $0.70(2.65)$ & -6 to 6 & $0.92(2.42)$ & -5 to 5 & $0.79(2.52)$ & -6 to 6 \\
\hline
\end{tabular}

Legend: $\mathrm{EPI}=$ electronic portal image; $\mathrm{DRR}=$ digitally reconstructed radiograph; $\mathrm{ML}=$ medial-lateral; $\mathrm{LM}=$ latero-medial; $\mathrm{AP}=$ anterior-posterior; $\mathrm{CC}=$ cranio-caudal; $\mathrm{LL}=$ latero-lateral. 
Table 2. Inter-rater (inter-observer) agreement for all patients $(n=40)$, based on Kappa statistics [13]

\begin{tabular}{|c|c|c|c|c|c|c|}
\hline & \multicolumn{2}{|c|}{ Radiation oncologist vs Senior therapist } & \multicolumn{2}{|c|}{ Radiation oncologist vs Junior therapist } & \multicolumn{2}{|c|}{ Senior technologist vs Junior therapist } \\
\hline & $\begin{array}{c}\text { Weighted } \\
\text { Cohen's Kappa }\end{array}$ & $95 \% \mathrm{CI}$ & $\begin{array}{c}\text { Weighted } \\
\text { Cohen's Kappa }\end{array}$ & $95 \% \mathrm{CI}$ & $\begin{array}{c}\text { Weighted } \\
\text { Cohen's Kappa }\end{array}$ & $95 \% \mathrm{CI}$ \\
\hline ML AP & 0.60 & $0.49-0.71$ & 0.80 & $0.73-0.87$ & 0.55 & $0.41-0.69$ \\
\hline ML CC & 0.55 & $0.42-0.72$ & 0.72 & $0.62-0.82$ & 0.46 & $0.32-0.60$ \\
\hline ML LL & 0.51 & $0.39-0.63$ & 0.58 & $0.48-0.68$ & 0.41 & $0.27-0.56$ \\
\hline LM AP & 0.79 & $0.69-0.88$ & 0.77 & $0.68-0.86$ & 0.68 & $0.56-0.81$ \\
\hline LM CC & 0.40 & $0.23-0.58$ & 0.75 & $0.66-0.84$ & 0.30 & $0.15-0.44$ \\
\hline LM LL & 0.84 & 0.74- 0.94 & 0.73 & $0.63-0.82$ & 0.35 & $0.18-0.52$ \\
\hline
\end{tabular}

Legend: $\mathrm{ML}=$ medial-lateral; $\mathrm{LM}=$ latero-medial; $\mathrm{AP}=$ anterior-posterior $; \mathrm{CC}=$ cranio-caudal; $\mathrm{LL}=$ latero-lateral; $\mathrm{CI}=$ confidence interval.

Based on the misalignment registered by a radiation oncologist, senior and junior technologist 0,1 and 0 patients would need further EPI verification (due to a misalignment with DRR $>5 \mathrm{~mm}$ ), respectively. The difference in measurements between observers (120 delta values) was 3, 4 and $5 \mathrm{~mm}$ in 4, 1 and 1 case, respectively. In all other 114 cases (95\%) the difference was $<2 \mathrm{~mm}$.

As far as LM projection is concerned, a mean AP EPI-DRR misalignment registered by a radiation oncologist, senior and junior technologist was $-2.15 \mathrm{~mm},-2.25 \mathrm{~mm}$ and $-2.17 \mathrm{~mm}$, respectively (tab. 1). Based on the misalignment reported by a radiation oncologist, senior and junior technologist 3 , 5 and 3 patients would need further EPI verification (due to a misalignment with DRR $>5 \mathrm{~mm}$ ), respectively. The difference in measurements between observers (120 delta values) was 3 and $4 \mathrm{~mm}$ in 3 and 1 case, respectively. In all other 116 cases $(96.7 \%)$ the difference was $<2 \mathrm{~mm}$.

A mean CC EPI-DRR misalignment on the LM projection registered by a radiation oncologist, senior and junior technologist was $1.5 \mathrm{~mm}, 1.65 \mathrm{~mm}$ and $1.4 \mathrm{~mm}$, respectively. Based on the misalignment reported by a radiation oncologist, senior and junior technologist 3,1 and 2 patients would need further EPI verification (due to a misalignment with DRR > $5 \mathrm{~mm}$ ), respectively. The difference in measurements between observers (120 delta values) was 3, 4, 5, 6, 8 and 9 mm 11, 4, $1,1,1$ and 1 case, respectively. In all other 101 cases (84\%) the difference was $<2 \mathrm{~mm}$.

A mean LL EPI-DRR misalignment on the LM projection registered by a radiation oncologist, senior and junior technologist were $0.75 \mathrm{~mm}, 0.7 \mathrm{~mm}$ and $0.92 \mathrm{~mm}$, respectively. Based on the misalignment registered by a radiation oncologist, senior and junior technologist 1, 0 and 1 patients would need further EPI verification (due to a misalignment with DRR $>5 \mathrm{~mm}$ ), respectively. The difference in measurements between observers (120 delta values) was 3 and $4 \mathrm{~mm}$ in 2 and 2 cases, respectively. In all other 116 cases $(96.7 \%)$ the difference was $<2 \mathrm{~mm}$.

When considering the whole set of 720 EPI-DRR comparisons, the difference between 6 and $10 \mathrm{~mm}$ (a threshold for the EPI repetition according to the institutional protocol) was found in 35 of comparisons (5\%) and no case requiring re-simulation (necessary if an EPI-DRR misalignment is > $10 \mathrm{~mm}$ ) was registered.

In $666(92.5 \%)$ out of all 720 delta values, the difference in measurements between observers was $<2 \mathrm{~mm}$. In the remaining 54 cases $(7.5 \%)$ a difference $>2 \mathrm{~mm}$ was registered, exhibiting values of $3 \mathrm{~mm}$ in 31 cases ( $4.3 \%), 4 \mathrm{~mm}$ in 11 cases (1.5\%), $5 \mathrm{~mm}$ in 8 cases (1.1\%), $6 \mathrm{~mm}$ in 2 cases $(0.2 \%), 8 \mathrm{~mm}$ in 1 case, $9 \mathrm{~mm}$ in 1 case $(0.1 \%)$.

Table 2 presents the weighted Cohen's Kappa values calculated for the above reported comparisons. High inter-observer agreement was found, it was almost perfect or substantial in 9 out of 18 comparisons ( 3 observers measuring 3 directions in 2 projections of EPI images) with weighted Cohen's Kappa $>0.61$.

When analyzed with regard to the health professional category, substantial inter-observer agreement was found between radiation oncologist and junior therapist $(0.75 \pm 0.07)$ and between radiation oncologist and senior therapist $(0.62$ $\pm 0.17)$; while moderate agreement $(0.46 \pm 0.14)$ was found between senior and junior therapists.

\section{Discussion}

Our study was based on a series of EPI set-up verification data coming from a population of 40 breast cancer patients, which were interpreted by 3 observers with different professional skills. The results account for an overall high accuracy in patient positioning and point out a consistent agreement among radiation oncologist and therapists evaluations. The EPI-DRR misalignment was $<5 \mathrm{~mm}$ in $95 \%$ of 720 comparisons. The in measurements between observers was $<2 \mathrm{~mm}$ in $92.5 \%$ of 720 delta values. These findings are surprisingly good considering the relatively low quality of the EPI images and therefore it is suggested to support the practise of entrusting trained therapists with EPI-based patient set-up verification in breast cancer radiation therapy.

Indeed, based on this study results, our institutional set-up verification protocol has been modified including therapist participation in the EPI evaluation in breast cancer. Further training and comparison analysis has been undertaken in order to extent the role of radiation therapists in 
the cone beam computer tomography-based image guided radiotherapy.

As far as patient set-up accuracy is concerned, our findings confirm previous studies, which reported high reproducibility and low inter- and intra-fractional set-up deviations in breast cancer radiotherapy $[14,15]$. In our series, when considering the whole set of evaluations performed by the 3 observers, detected EPI-DRR misalignments along AP, CC and LL directions were (mean \pm standard deviation) $-2.1 \pm$ $2.5,1.5 \pm 2.8,0.7 \pm 2.3 \mathrm{~mm}$, respectively, with an overall average variability of $2.5 \mathrm{~mm}$. Highest set-up uncertainties were found along the AP direction, with values compatible with those induced by respiratory motion on the thoracic region [12]. These results attest that the positioning procedure and the use of patient support devices (inclined plate with arm rests) allow employing the $5 \mathrm{~mm}$ safety CTV-PTV margin in the treatment planning [17]. Nevertheless, the detection of isolated misalignments up to $9 \mathrm{~mm}$ suggests a need for more effective technologies in selected patient set-up verification, applicable at every treatment fraction, such as non-ionizing infrared optical tracking systems $[18,19]$.

In daily radiotherapy clinical practise, EPI represents a widely applied approach to patient set-up verification. Despite the lack of specific studies focused on the agreement among radiation oncologists and therapists in EPI-based set-up quality evaluation, in several departments this task is entrusted to trained radiation therapists [14, 20, 21]. Interrater variability is a well-known issue in radiation therapy that affect both the treatment planning and the patient setup verification phases [1]. Wide variability in set-up quality reviews performed by physicians has been reported and related to observer's training and experience $[5,22,23]$. Beside the specific observer's skills, inter-rater variability depends to a large extent upon the presence of clearly identifiable anatomical landmarks (which varies as a function of the specific targeted anatomical area) and image quality. This latter emerges from the specific EPI equipment, from the available software for image processing, analysis and representation and from the specific procedure used for image registration (manual, automatic).

We are aware of the limitations of our study, including the use of on-line registration as reference data and low number of observers. One can argue that on-line EPI registration might be biased by limited time available (the patient is on the couch during evaluation). Indeed, in several institutions, the introduction of a new observer is based on the off-line verification by an experienced observer. Last but not least, the use of Kappa statistics may also be criticized, due to its known dependence of prevalence of the observed condition, low reproducibility and difficult interpretation in clinical practice (i.e. in case of our study, what level of agreement is necessary before trained therapist is allowed to perform on-line EPI registration?) $[24,25]$.

Despite these limitations, we think that our study might be of value for the radiation oncologists, medical physicists, radiation therapists and other professionals dealing with breast cancer patients treated with radiotherapy. Our results showing an overall good agreement between physicians and trained therapists might serve as a measure for the optimization of the workload in busy radiotherapy departments. The study is specific for breast cancer EPI-based evaluation, which benefits from the presence in the EPI images and corresponding DRRs of the easily identifiable bony landmarks and geometrical references (thoracic wall, lung margins, air gap). In this scenario, we believe that the professional skills of properly trained therapists to perform routine verification procedures based on EPI are adequate, provided that they rely on consultancy with radiation oncologists and/or medical physicists for facing off-nominal situations. In daily clinical practice, this provides a safe alternative strategy, as a way to optimize quality control procedures and optimize the workload of radiotherapy departments featuring high patient throughput. Evidence from radiology studies shows that, with appropriate education and training, the accuracy of therapists in interpreting plain diagnostic images is comparable to that of radiologists [26]. Extending the role of therapists in a specific range of imaging tasks may help meet demand, relieving some pressure on clinicians $[26,27]$. In consequence, these types of studies are helpful in department workload optimization and might allow wider adoption and implementation of complex and evolving radiotherapy technologies.

\section{References}

[1] GENOVESI D, CEFARO GA, VINCIGUERRA A, AUGURIO A, DI TOMMASO M, et al. Interobserver variability of clinical target volume delineation in supra-diaphragmatic Hodgkin's disease: a multi-institutional experience. Strahlenther Onkol 2011; 187: 357-66. http://dx.doi.org/10.1007/s00066-0112221-y

[2] LIEBICH J, LICHER J, SCHERF C, KARA E, KOCH N, et al. Simple proposal for dosimetry with an Elekta iViewGT ${ }^{\text {tm }}$ electronic portal imaging device (EPID) using commercial software modules. Strahlenther Onkol 2011; 187: 316-21. http://dx.doi.org/10.1007/s00066-011-2176-Z

[3] PECHARROMAN-GALLEGO R, MANS A, SONKE JJ, STROOM JC, OLACIREGUI-RUIZ I, et al. Simplifying EPID dosimetry for IMRT treatment verification. Med Phys 2011; 38: 983-92. http://dx.doi.org/10.1118/1.3547714

[4] RICHTER A, SWEENEY R, BAIER K, FLENTJE M, GUCKENBERGER M. Effect of breathing motion in radiotherapy of breast cancer: $4 \mathrm{D}$ dose calculation and motion tracking via EPID. Strahlenther Onkol 2009; 185: 425-30. http://dx.doi. org/10.1007/s00066-009-1980-1

[5] KRUSE JJ, HERMAN MG, HAGNESS CR, DAVIS BJ, GARCES YI, et al. Electronic and film portal images: A comparison of landmark visibility and review accuracy. Int J Radiat Oncol Biol Phys 2002; 54: 584-91. http://dx.doi.org/10.1016/S03603016(02)02955-3

[6] BODA-HEGGEMANN J, LOHR F, WENZ F, FLENTJE M, GUCKENBERGER M. kV cone-beam CT-based IGRT: 
a clinical review. Strahlenther Onkol 2011; 187: 284-91. http://dx.doi.org/10.1007/s00066-011-2236-4

[7] BUDACH W, BOLKE E, FIETKAU R, BUCHALI A, WENDT TG, et al. Evaluation of time, attendance of medical staff, and resources during radiotherapy for head and neck cancer patients: The DEGRO-QUIRO Trial. Strahlenther Onkol 2011; 187: 449-60. http://dx.doi.org/10.1007/s00066-011-2273-Z

[8] ZUCCA S, CARAU B, SOLLA I, GARIBALDI E, FARACE P, et al. Prostate image-guided radiotherapy by megavolt conebeam CT. Strahlenther Onkol 2011; 187: 473-8. http://dx.doi. org/10.1007/s00066-011-2241-7

[9] ICRU. Report 62: Prescribing, recording and reporting photon beam therapy (supplement to ICRU report 50). Bethesda: International Commission on Radiation Units and Measurements. 1999.

[10] FERRARI A, LEONARDI MC, IVALDI GB, FANTI P, DELLACQUA V, et al. Clinical volumes in breast cancer: First toxicity results of prospective randomized study comparing hypofractionated vs standard radiotherapy regimens. Tumori 2010; 10(Suppl): s27.

[11] IVALDI GB, LEONARDI MC, ORECCHIA R, ZERINI D, MORRA A, et al. Preliminary results of electron intraoperative therapy boost and hypofractionated external beam radiotherapy after breast-conserving surgery in premenopausal women. Int J Radiat Oncol Biol Phys 2008; 72: 485-93. http://dx.doi. org/10.1016/j.ijrobp.2007.12.038

[12] BARONI G, GARIBALDI C, SCABINI M, RIBOLDI M, CATALANO G, et al. Dosimetric effects within target and organs at risk of inter-fractional patient mispositioning in left breast cancer radiotherapy. Int J Radiat Oncol Biol Phys 2004;59:86171. http://dx.doi.org/10.1016/j.ijrobp.2004.02.043

[13] LANDIS JR, KOCH GG. The measurement of observer agreement for categorical data. Biometrics 1977: 33: 159-74. http://dx.doi.org/10.2307/2529310

[14] HURKMANS CW, REMEIJER P, LEBESQUE JV, MIJNHEER BJ. Set-up verification using portal imaging; review of current clinical practice. Radiother Oncol 2001; 58: 105-20. http:// dx.doi.org/10.1016/S0167-8140(00)00260-7

[15] BOHMER D, FEYER P, HARDER C, KORNER M, STERNEMANN M, et al. Verification of set-up deviations in patients with breast cancer using portal imaging in clinical practice. Strahlenther Onkol 1998; 174(Suppl 2): 36-9.

[16] TOPOLNJAK R, SONKE JJ, NIJKAMP J, RASCH C, MINKEMA D, et al. Breast patient setup error assessment: comparison of electronic portal image devices and conebeam computed tomography matching results. Int J Radiat
Oncol Biol Phys 2010; 78: 1235-43. http://dx.doi.org/10.1016/ j.ijrobp.2009.12.021

[17] VAN HERK M. Errors and margins in radiotherapy. Semin Radiat Oncol 2004; 14: 52-64. http://dx.doi.org/10.1053/ j.semradonc.2003.10.003

[18] SPADEA MF, BARONI G, RIBOLDI M, TAGASTE B, GARIBALDI C, et al. Patient set-up verification by infrared optical localization and body surface sensing in breast radiation therapy. Radiother Oncol 2006;79:170-8. http://dx.doi. org/10.1016/j.radonc.2006.02.011

[19] SCHOFFEL PJ, HARMS W, SROKA-PEREZ G, SCHLEGEL W, KARGER CP. Accuracy of a commercial optical 3D surface imaging system for realignment of patients for radiotherapy of the thorax. Phys Med Biol 2007; 52: 3949-63. http://dx.doi. org/10.1088/0031-9155/52/13/019

[20] BERTHELETE, TRUONG PT,ZAVGORODNIS, MORAVAN V, LIU MC, et al. Consistency in electronic portal imaging registration in prostate cancer radiation treatment verification. Radiat Oncol 2006; 1: 37. http://dx.doi.org/10.1186/1748717X-1-37

[21] VAN LIN EN, VAN DER VIGHT L, HIUZENGA H, KAANDERS JH, VISSER AG. Set-up improvement in head and neck radiotherapy using a $3 \mathrm{D}$ off-line EPID-based correction protocol and a customised head and neck support. Radiother Oncol 2003: 68: 137-48. http://dx.doi.org/10.1016/ S0167-8140(03)00134-8

[22] PERERA T, MOSELEY J, MUNO P. Subjectivity in interpretation of portal films. Int J Radiat Oncol Biol Phys 1999; 45: 529-34. http://dx.doi.org/10.1016/S0360-3016(99)00204-7

[23] LEWIS DG, RYAN KR, SMITH CW. Observer variability when evaluating patient movement from electronic portal images of pelvic radiotherapy fields. Radiother Oncol 2005; 74: 275-81. http://dx.doi.org/10.1016/j.radonc.2004.11.009

[24] SIERRA F, CARDENAS A. Evidence-based medicine (EBM) in practice: agreement between observers rating esophageal varices: how to cope with chance? Am J Gastroenterol 2007; 102: 2363-6. http://dx.doi.org/10.1111/j.1572-0241.2007.01225.x

[25] VIERA A, GARRETT J. Understanding Interobserver Agreement:The Kappa Statistic. Fam Med 2005; 37: 360-3.

[26] SMITH TN, BAIRD M. Radiographers' role in radiological reporting: a model to support future demand. Med J Aust 2007; 186: 629-31.

[27] WHITE E, KANE G. Radiation medicine practice in the image-guided radiation therapy era: new roles and new opportunities. Semin Radiat Oncol 2007; 17: 298-305. http://dx.doi. org/10.1016/j.semradonc.2007.07.001 